Pacific Journal of Mathematic

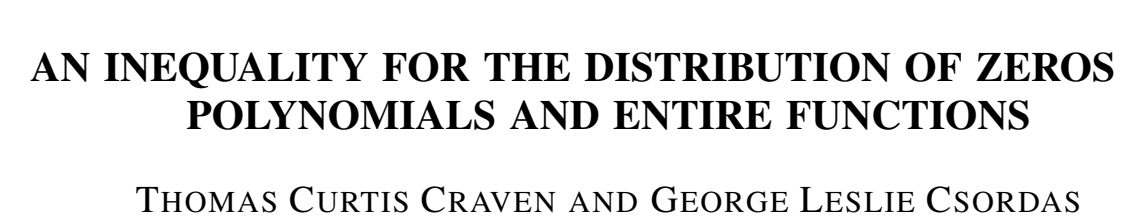




\section{AN INEQUALITY FOR THE DISTRIBUTION OF ZEROS OF POLYNOMIALS AND ENTIRE FUNCTIONS}

\section{Thomas Craven and George Csordas}

An inequality is established which provides a unifying principle for the distribution of zeros of real polynomials and certain entire functions. This inequality extends the applicability of multiplier sequences to the class of all real polynomials. The various consequences obtained generalize and supplement several results due to Hermite-Poulain, Laguerre, Marden, Obreschkoff, Polya and Schur.

1. Introduction. In the vast literature dealing with the distribution of zeros of real polynomials and real entire functions, an important role is played by linear transformations $T$ which possess the following property:

$$
Z_{c}(T[f]) \leqq Z_{c}(f),
$$

where $f$ is a polynomial and $Z_{c}(f)$ denotes the number of nonreal zeros of $f$, counting multiplicities. If $T=D=d / d x$, then the above inequality is a consequence of Rolle's theorem. If $h$ is a real polynomial with only real zeros and $T=h(D)$, then (1) follows from the classical Hermite-Poulain theorem [12, p. 4]. There are many other linear transformations $T$ which satisfy inequality (1). Indeed, let $\Gamma=\left\{\gamma_{k}\right\}_{k=0}^{\infty}$ be a sequence of real numbers and for an arbitrary real polynomial $f(x)=\sum_{k=0}^{n} a_{k} x^{k}$ define $\Gamma[f]$ by

$$
\Gamma[f(x)]=\sum_{k=0}^{n} a_{k} \gamma_{k} x^{k} .
$$

If $\Phi(x)=\sum a_{k} x^{k}$ is a real entire function, then set $\Gamma[\Phi(x)]=\sum a_{k} \gamma_{k} x^{k}$, whenever this series converges. Now let $Q(x)$ be a real polynomial with only real negative zeros. Let $\Gamma=\{Q(k)\}_{k=0}^{\infty}$. Then Laguerre's theorem [12, p. 6] asserts that

$$
\begin{aligned}
Z_{C}(\Gamma[f]) & =Z_{c}\left(\sum_{k=0}^{n} a_{k} Q(k) x^{k}\right) \\
& \leqq Z_{C}(f),
\end{aligned}
$$

where $f(x)=\sum_{k=0}^{n} a_{k} x^{k}$ is an arbitrary real polynomial.

The real sequences $\Gamma=\left\{\gamma_{k}\right\}$ for which $\Gamma[f]$ has only real zeros whenever $f$ is a real polynomial with only real zeros, have been completely characterized by Pólya and Schur in their celebrated paper [20] entitled, Über zwei Arten von Faktorenfolgen in der 
Theorie der algebraischen Gleichungen. In this paper Pólya and Schur called a sequence $\Gamma=\left\{\gamma_{k}\right\}_{k=0}^{\infty}$ of real numbers a multiplier. sequence of the first kind if $\Gamma$ takes every real polynomial $f(x)$ which has only real zeros into a polynomial $\Gamma[f(x)]$ (defined by (2)) of the same class. These authors termed a sequence $\Gamma=\left\{\gamma_{k}\right\}_{k=0}^{\infty}$ of real numbers a multiplier sequence of the second kind if $\Gamma$ takes every real polynomial $f(x)$, all whose zeros are real and of the same sign, into a polynomial all of whose zeros are real. Thus, with this nomenclature, the aforementioned theorem of Laguerre states, in particular, that the sequence $\Gamma=\{Q(k)\}_{k=0}$ is a multiplier sequence of the first kind. We hasten to add that there are several other multiplier sequences of the first kind which are known to enjoy inequality (1) (see, for example, [12, Satz 5.8, Satz 5.13 and Satz 5.14]).

In $\S 2$ we shall introduce a new family of multiplier sequences of the first kind which depend continuously on a parameter $t$ (Theorem 2). With the aid of these sequences and one of our previous results (Theorem 1), we shall completely characterize all real sequences $\Gamma=\left\{\gamma_{k}\right\}$ which satisfy the inequality

$$
Z_{c}(\Gamma[f]) \leqq Z_{c}(f)
$$

where $f$ is an arbitrary real polynomial and where $\Gamma[f]$ is defined by (2) (Corollary 4). Indeed, our main result, the fundamental inequality, (we will use this epithet to distinguish inequality (3) from the numerous inequalities that the reader will encounter in the sequel) asserts that if $\Gamma$ is a multiplier sequence of the first kind, then inequality (3) holds for all real polynomials $f$ (Theorem 3). We shall also discuss conditions when strict inequality holds in (3) (Theorem 4 and Theorem 5). At the end of this section, we shall show that, in a certain sense, inequality (3) is best possible.

Section 3 is devoted to several applications and consequences of the fundamental inequality. Indeed, the various corollaries in this section demonstrate that inequality (3) serves as a unifying principle for many results of the type we cited above. In particular, in this section we shall extend the theorems of Laguerre (Corollary 11), Hermite-Poulain (Corollary 14), Pólya and Schur (Corollary 7), Pólya (Corollary 8 and Corollary 9) and Schur (Corollary 12).

2. The fundamental inequality. In [20] Pólya and Schur provided both algebraic and transcendental characterizations of multiplier sequences. We begin this section with a brief review of the transcendental characterizations of these sequences. Let $\Gamma=\left\{\gamma_{k}\right\}_{k=0}^{\infty}$ be a sequence of real numbers. Then in order that $\Gamma$ 
be a multiplier sequence of the first kind, it is necessary and sufficient that the series $\Phi(x)=\sum\left(\gamma_{k} / k !\right) x^{k}$ converge in the entire plane, and that the entire function $\Phi(x)$ or $\Phi(-x)$ can be represented in the form

$$
\Phi(x)=c e^{o x} x^{m} \prod_{n=1}^{\infty}\left(1+\frac{x}{x_{n}}\right),
$$

where $\sigma \geqq 0, \quad x_{n}>0, \quad c \in R, \sum_{n=1}^{\infty} x_{n}^{-1}<\infty$ and $m$ is a nonnegative integer. In order that the sequence $\Gamma$ be a multiplier sequence of the second kind, it is necessary and sufficient that the series $\Phi(x)=$ $\sum\left(\gamma_{k} / k !\right) x^{k}$ converge in the entire plane, and that the entire function $\Phi(x)$ can be represented in the form

$$
\Phi(x)=c e^{-\alpha x^{2}+\beta x} x^{m} \prod_{n=1}^{\infty}\left(1-\frac{x}{x_{n}}\right) e^{x / x_{n}},
$$

where $\alpha \geqq 0, c, \beta$ and $x_{n}$ are real, $\sum_{n=1}^{\infty} x_{n}^{-2}<\infty$ and $m$ is a nonnegative integer. In the sequel we will adhere to the following nomenclature. A real entire function $\Phi$ is called a function of type II in the Laguerre-Pólya class if it admits a representation of the form (5). Entire functions which admit a representation of the form (4) are termed functions of type $I$ in the Laguerre-Pólya class. The significance of the Laguerre-Pólya class in the theory of entire functions (Levin [7, Chapter 8]) is natural since Pólya [13] has shown that functions of the type II, and only those, are the uniform limits, on compact subsets of the plane, of polynomials with only real zeros.

The inequality we shall establish in Theorem 3 may be derived from any one of several results that the authors obtained in connection with their investigations of the structure of certain real algebraic curves. In the present setting, it will be fruitful to use the following theorem that we proved in [3].

THeOREM 1. Let $h(x)=\sum_{0}^{n} b_{k} x^{k}$ be a real polynomial with only real nonpositive zeros and let $f(x)$ be an arbitrary real polynomial. Then

$$
Z_{c}\left(\sum_{0}^{n} b_{k} x^{k} f^{(k)}(x)\right) \leqq Z_{c}(f)
$$

We remark that in the special case when $f$ has only real zeros this theorem was first proved by Pólya and Schur [20, p. 107] (see also Pólya [15] or [1]). With the aid of Theorem 1, we shall show below that a certain family of sequences which depend continuously on a parameter $t$ is a family of multiplier sequences of the first 
kind.

In order to facilitate our description of these sequences, we require some additional notation and terminology. For an arbitrary real polynomial $f(x)$ of degree $n$, we define $f^{*}(x) \equiv x^{n} f\left(x^{-1}\right)$. If $m$ is a positive integer, we set

$$
\begin{gathered}
J_{m}=\left\{1,1,1-\frac{1}{m}, \cdots,\left(1-\frac{1}{m}\right)\left(1-\frac{2}{m}\right) \cdots\left(1-\frac{m-1}{m}\right),\right. \\
0,0 \cdots\} .
\end{gathered}
$$

Then $J_{m}$, also known as the multiplier sequence of Jensen, is a multiplier sequence of the first kind (see Levin [7]). Hence, if $\Gamma=\left\{\gamma_{k}\right\}, \gamma_{k} \geqq 0$, is a multiplier sequence of the first kind, then it follows that the zeros of the polynomials

$$
g_{k}(t) \equiv\left(\Gamma\left[(1+t)^{k}\right]\right), k=0,1,2, \cdots,
$$

and

$$
\begin{aligned}
g_{k, m}^{*}(t) & \equiv\left(J_{m}\left[g_{k}(t)\right]\right)^{*} \\
& =\sum_{j=0}^{\mu}\left(1-\frac{1}{m}\right) \cdots\left(1-\frac{j-1}{m}\right) \gamma_{j}\left(\begin{array}{l}
k \\
j
\end{array}\right) t^{k-j},
\end{aligned}
$$

where $\mu=\min (k, m)$, are all real and nonpositive. We also note that if $\Phi(x)=\Gamma\left[e^{x}\right]$, so that $\Phi(x)$ is a function of type $I$ in the Laguerre-Pólya class, then the polynomials $g_{k}^{*}$ are generated by $e^{x t} \Phi(x)$; that is,

$$
e^{x t} \Phi(x)=\sum_{k=0}^{\infty} g_{k}^{*}(t) \frac{x^{k}}{k !} .
$$

But for a fixed $t>0, e^{x t} \Phi(x)$ is also a function of type $I$ in the Laguerre-Pólya class. Therefore, by the aforementioned transcendental characterizations of multiplier sequences, for each fixed $t_{0}>0$, the sequence $\left\{g_{k}^{*}\left(t_{0}\right)\right\}_{k=0}^{\infty}$ is a multiplier sequence of the first kind.

Preliminaries aside, we shall now prove that for each positive integer $m$ the sequence $\left\{g_{k, m}^{*}\left(t_{0}\right)\right\}_{k=0}^{\infty}, t_{0}>0$, constructed above satisfies inequality (3).

THEOREM 2. Let $\Gamma=\left\{\gamma_{k}\right\}_{k=0}^{\infty}, \gamma_{k} \geqq 0$, be a multiplier sequence of the first kind. For each positive integer $m$ let $\Lambda_{t, m}=\left\{g_{k, m}^{*}(t)\right\}$. Let $f(x)=\sum_{k=0}^{n} a_{k} x^{k}$ be an arbitrary real polynomial of degree $n$. Then for each fixed $t>0$,

$$
Z_{c}\left(\Lambda_{t, m}[f]\right) \leqq Z_{C}(f)
$$


In particular $\Lambda_{t, m}$ is a multiplier sequence of the first kind.

Proof. It suffices to consider the case when possibly some but not all of the terms of $\Gamma=\left\{\gamma_{k}\right\}$ are zero. Since $\Gamma$ is a multiplier sequence of the first kind the relations $\gamma_{j} \gamma_{l} \neq 0$ and $\gamma_{k}=0$ for any $k, j<k<l$, cannot hold at the same time (see, for example, Craven and Csordas [2, Theorem 3.4(b), p. 807]). Furthermore, we note that if $\gamma_{0}=\gamma_{1}=\cdots=\gamma_{p}=0, \gamma_{p+1} \neq 0$ and $m \leqq p$, then $\Lambda_{t, m}[f] \equiv 0$. Thus, if $\Gamma$ has precisely $p+1$ leading zero terms, then, to avoid trivialities, we let $m \geqq p+1$.

Now we set

$$
\begin{aligned}
g_{m}(t) & =\Gamma\left[(1+t)^{m}\right] \\
& =\Sigma\left(\begin{array}{l}
m \\
k
\end{array}\right) \gamma_{k} t^{k}
\end{aligned}
$$

and observe that the degree of $g_{m}(t)$ need not be $m$. If $D_{t}$ denotes differentiation with respect to $t$, then for each fixed $x$

$$
g_{m}\left(D_{t}\right) f(x t)=\sum\left(\begin{array}{c}
m \\
k
\end{array}\right) \gamma_{k} x^{k} f^{(k)}(x t) .
$$

Thus, for a fixed but arbitrary $t_{0}>0$, we obtain

$$
\left[g_{m}\left(D_{t}\right) f(x t)\right]_{t=t_{0}}=\sum\left(\begin{array}{c}
m \\
k
\end{array}\right) \gamma_{k} x^{k} f^{(k)}\left(x t_{0}\right) \text {. }
$$

If we set $y=x t_{0}$, then

$$
\sum\left(\begin{array}{c}
m \\
k
\end{array}\right) \gamma_{k} x^{k} f^{(k)}\left(x t_{0}\right)=\sum\left(\begin{array}{c}
m \\
k
\end{array}\right) \gamma_{k} t_{0}^{-k} y^{k} f^{(k)}(y)
$$

Since $\Gamma$ is a multiplier sequence of the first kind, the polynomial $\sum\left(\begin{array}{c}m \\ k\end{array}\right) \gamma_{k} t_{0}^{-k} y^{k}$ has only real nonpositive zeros. Therefore, we may invoke Theorem 1 and conclude that

$$
Z_{C}\left(\sum\left(\begin{array}{c}
m \\
k
\end{array}\right) \gamma_{k} t_{0}^{-k} y^{k} f^{(k)}(y)\right) \leqq Z_{C}(f)
$$

and consequently that

$$
Z_{C}\left(\left[g_{m}\left(D_{t}\right) f(x t)\right]_{t=t_{0}}\right) \leqq Z_{C}(f) .
$$

Now a computation shows that

$$
\left[g_{m}\left(D_{t}\right) f(x t)\right]_{t=t_{0}}=\sum_{k=0}^{n} a_{k} x^{k} \sum_{j=0}^{\mu}\left(\begin{array}{c}
k \\
j
\end{array}\right) \gamma_{j} \frac{m !}{(m-j) !} t_{0}^{k-j},
$$


where $\mu=\min (k, m)$. In this formulation we added the stipulation that $\mu=\min (k, m)$ since we allow the positive integer $m$ to be less than the degree of $f$. If we replace in the right-hand side of the expression $t_{0}$ by $t_{0} m$ and $x$ by $m^{-1} x$, then the inequality on the number of nonreal zeros is preserved and thus we obtain

$$
Z_{C}\left(\sum_{k=0}^{n} a_{k} x^{k} \sum_{j=0}^{\mu}\left(\begin{array}{c}
k \\
j
\end{array}\right) \gamma_{j} \frac{m !}{(m-j) ! m^{j}} t_{0}^{k-j}\right) \leqq Z_{C}(f),
$$

where $\mu=\min (k, m)$. That is, for each fixed $t>0$,

$$
Z_{C}\left(\Lambda_{t, m}[f]\right) \leqq Z_{C}(f) \text {. }
$$

Finally, if $f$ has only real zeros, then the above inequality implies that $\Lambda_{t, m}[f]$ has also only real zeros. In particular, the sequence $\Lambda_{t, m}$ is a multiplier sequence of the first kind. This completes the proof of Theorem 2.

As a consequence of Theorem 2, we obtain the following fundamental inequality.

THEOREM 3 (The Fundamental Inequality). Let $\Gamma=\left\{\gamma_{k}\right\}_{k=0}^{\infty}$ be a multiplier sequence of the first kind and let $f(x)=\sum_{k=0}^{n} a_{k} x^{k}$ be an arbitrary real polynomial of degree $n$. Then

$$
Z_{c}(\Gamma[f]) \leqq Z_{C}(f)
$$

Proof. First we assume that $\gamma_{k} \geqq 0$ for $k=0,1,2, \cdots$. Then by Theorem 2 for each positive integer $m, m>n$ and for each $t_{0}>0$ the following inequality holds

$$
Z_{C}\left(\sum_{k=0}^{n} a_{k} x^{k} \sum_{j=0}\left(\begin{array}{l}
k \\
j
\end{array}\right) \gamma_{j} \frac{m !}{(m-j) ! m^{j}} t_{0}^{k-j}\right) \leqq Z_{C}(f) .
$$

Now we take the limit as $m \rightarrow \infty$ followed by the limit as $t_{0} \rightarrow 0$. Under these limiting processes the above inequality prevails by Hurwitz's theorem and thus we conclude that if $\gamma_{k} \geqq 0$, then

$$
Z_{C}(\Gamma[f]) \leqq Z_{C}(f)
$$

Since $\Gamma=\left\{\gamma_{k}\right\}$ is a multiplier sequence of the first kind the terms $\gamma_{k}$ either all have the same sign or they have alternating signs. In the latter case we apply the above argument to the sequence $\left\{(-1)^{k+i} \gamma_{k}\right\}_{k=0}^{\infty}$, where $i=0$ if $\gamma_{2 k} \geqq 0$ and $i=1$ if $\gamma_{2 k+1} \geqq 0$. Thus, we see that inequality (6) remains valid if the terms $\gamma_{k}$ have alternating signs and so the proof of the theorem is complete. 
REMARK. If $\Gamma=\left\{\gamma_{k}\right\}$ is a multiplier sequence of the first kind, then the terms $\gamma_{k}$ either all have the same sign or they have alternating signs. For reasons of convenience we shall often assume in the sequel that $\gamma_{k} \geqq 0$ for all $k$. Indeed, if $\Phi(x)=\Gamma\left[e^{x}\right]$ is a function of type $I$ in the Laguerre-Pólya class, then so is the function $\Phi(-x)$.

The significance of inequality (6) is, in part, due to the fact that it extends the applicability of multiplier sequences of the first kind to the class of all real polynomials. In particular, we have solved here the following problem: characterize all real sequences $\Gamma=\left\{\gamma_{k}\right\}$ which satisfy inequality (6) for all real polynomials $f$. The solution to this problem is summarized in the following corollary.

COROLLARY 4. Let $\Gamma=\left\{\gamma_{k}\right\}$ be a sequence of real numbers. Then $\Gamma$ is a multiplier sequence of the first kind if and only if for any real polynomial $f$

$$
Z_{C}(\Gamma[f]) \leqq Z_{c}(f) .
$$

In dealing with inequalities it is always important to know when or under what conditions on inequality of the form " $\leqq$ " can reduce to an equality. A complete specification of the conditions under which equality holds in (6) seems to be difficult since this inequality depends both on the multiplier sequence $\Gamma$ and on the polynomial $f$. On the other hand, if $\Gamma$ is of a particularly simple form, as for example is the case if $\Gamma=\left\{1, r, r^{2}, \cdots\right\}, r \neq 0$, then clearly for any real polynomial $f, Z_{c}(\Gamma[f])=Z_{c}(f)$. Another problem of interest is to characterize multiplier sequences $\Gamma$ and polynomials $f$ for which $Z_{c}(\Gamma[f])=0$, when $f$ possesses some nonreal zeros. The following two theorems provide a partial solution to the above cited problems.

THEOREM 5 (See Obreschkoff [12, p. 126]). Let $f(x)=\sum_{k=0}^{n} a_{k} x^{k}$ be a real polynomial with zeros $z_{1}, \cdots, z_{n}$. Suppose for some nonnegative integer $p, 0 \leqq p \leqq n$,

$$
\left|\arg z_{k}\right|<\frac{\pi}{2 n+2-p}, k=1, \cdots, p,
$$

and that the remaining zeros of $f$, if any, satisfy

$$
\left|\arg z_{k}-\pi\right|<\frac{\pi}{n+p+2} .
$$

Let $\Gamma=\left\{\gamma_{k}\right\}, \gamma_{k}>0$, be a multiplier sequence of the first kind and 
let $\Lambda=\left\{\left(\begin{array}{l}n \\ k\end{array}\right) \gamma_{k}\right\}$. Then $\Lambda$ is a multiplier sequence of the first kind and

$$
Z_{c}(\Lambda[f])=0
$$

Proof. It is easy to see that if $\left\{\gamma_{k}\right\}$ and $\left\{\gamma_{k}^{\prime}\right\}$ are any two multiplier sequences of the first kind, then the composite sequence $\left\{\gamma_{k} \gamma_{k}^{\prime}\right\}$ is also a multiplier sequence of the first kind. Thus, if we compose the multiplier sequence of Jensen, $J_{n}$, with the sequence $\left\{n^{k}\right\}_{k}$ and then compose this resulting sequence with $\left\{\gamma_{k} / k !\right\}$ we obtain 1. Therefore, we conclude that $\Lambda$ is a multiplier sequence of the first kind. Since the zeros of the polynomial $\Gamma\left[(1+x)^{n}\right]$ are all real and negative, the conclusion that $Z_{C}(\Lambda[f])=0$ now follows from Obreschkoff's theorem [12, p. 126].

Our next theorem brings into a sharper focus the dependence of the fundamental inequality (6) on the multiplier sequence $\Gamma$ and on the polynomial $f$.

THeOREM 6. Let $\Phi(x)=\sum\left(\gamma_{k} / k !\right) x^{k}$ be an entire function of type I in the Laguerre-Pólya class and suppose that $\Phi$ has an infinite number of zeros. Let $\Gamma=\left\{\gamma_{k}\right\}$ and let $f(x)=\sum_{k=0}^{n} a_{k} x^{k}$ be a real polynomial of degree $n$. Then there exists a constant $K=$ $K(\Gamma, f)$, which depends on $\Gamma$ and $f$, such that for all real $\alpha,|\alpha|>K$

$$
Z_{c}(\Gamma[f(x+\alpha)])=0 .
$$

Proof. We may assume, without loss of generality, that $\gamma_{k}>0$ for all $k$. For each positive integer $m$, let $g_{m}(x)=\Gamma\left[(1+x)^{m}\right]$. Then the hypotheses about $\Phi$ imply that $g_{m}(x)$ has only real, simple zeros (see Csordas and Williamson [5]). Next, a simple calculation shows that for $\alpha$ real, $\alpha \neq 0$,

$$
\begin{aligned}
\Gamma[f(x+\alpha)] & =\sum_{k=0}^{n} \frac{f^{(k)}(\alpha)}{k !} \gamma_{k} x^{k} \\
& =\sum_{k=0}^{n} a_{k} \alpha^{k} g_{k}\left(\frac{x}{\alpha}\right) .
\end{aligned}
$$

Thus for $\alpha \neq 0$, the polynomials $P(x)=\sum_{k=0}^{n} a_{k} \alpha^{k} g_{k}(x)$ and $\Gamma[f(x+\alpha)]$ have the same number of real zeros. We now select $n+1$ real numbers $t_{0}, \cdots, t_{n}$ such that $t_{0}>t_{1}>\cdots>t_{n}, g_{n}^{\prime}\left(t_{j}\right)=0$ for $j=$ $1, \cdots, n-1, g_{n}\left(t_{0}\right)>0$ and $(-1)^{n} g_{n}\left(t_{n}\right)>0$. This is possible since the zeros of $g_{n}$ are all real and simple. Let

$$
M=\left(\max _{j} \sum_{k=0}^{n-1}\left|g_{k}\left(t_{j}\right)\right|\right)\left(\min _{j}\left|g_{n}\left(t_{j}\right)\right|\right)^{-1}
$$


and let

$$
K=\max \left(M \max _{k}\left|\frac{a_{k}}{a_{n}}\right|, 1\right) .
$$

Then for $|\alpha|>K$ the following estimates hold:

$$
\begin{aligned}
& \left|\sum_{k=0}^{n-1} a_{k} \alpha^{k} g_{k}\left(t_{j}\right)\right| \leqq|\alpha|^{n-1} \sum_{k=0}^{n-1}\left|a_{k} g_{k}\left(t_{j}\right)\right| \\
& \quad \leqq|\alpha|^{n-1}\left|a_{n}\right|\left(\max _{k}\left|\frac{a_{k}}{a_{n}}\right|\right)\left(\max _{j} \sum_{k=0}^{n-1}\left|g_{k}\left(t_{j}\right)\right|\right) \\
& \quad<|\alpha|^{n-1}\left[\frac{|\alpha|}{M \max _{k}\left|\frac{a_{k}}{a_{n}}\right|}\right]\left|a_{n}\right|\left[\max _{k}\left|\frac{a_{k}}{a_{n}}\right|\right]\left[\max _{j} \sum_{k=0}^{n-1}\left|g_{k}\left(t_{j}\right)\right|\right] \\
& \quad \leqq|\alpha|^{n}\left|a_{n}\right|\left(\min _{j}\left|g_{n}\left(t_{j}\right)\right|\right) \\
& \quad \leqq\left|\alpha^{n} a_{n} g_{n}\left(t_{j}\right)\right| .
\end{aligned}
$$

The strict inequality implies that $P\left(t_{j}\right)$ and $a_{n} \alpha^{n} g_{n}\left(t_{j}\right)$ have the same sign for each $j$. Thus, $P(x)$ has $n$ sign changes and a fortiori $n$ real roots for $|\alpha|>K$. But then $Z_{C}(\Gamma[f(x+\alpha)])=0$ for $|\alpha|>K$.

At this point it should be noted that while the linear operators $\Gamma$ and $D=d / d x$ enjoy many similar properties (see, for example, Corollaries 9 and 14 below), $\Gamma$, in general, is not translation invariant. In fact, simple (although somewhat laborious) examples show that if $\Gamma$ is a multiplier sequence of the first kind, then, in general,

$$
Z_{C}(\Gamma[f(x+\alpha)]) \neq Z_{C}(\Gamma[f]) .
$$

The elusive character of multiplier sequences $\Gamma$ of the first kind is further underscored by examples which show that, in general,

$$
Z_{c}(\Gamma[f]) \neq Z_{c}\left(\Gamma\left[f^{*}\right]\right),
$$

where $f^{*}(x)=x^{n} f(1 / x)$ and $f(x)$ is a real polynomial of degree $n$.

In the remainder of this section, we shall demonstrate that in a certain sense inequality (6) is best possible. If $\Gamma$ is a multiplier sequence of the second kind and if $f$ is a polynomial with only real nonpositive zeros, then Pólya has shown that $Z_{c}(\Gamma[f])=0$. In the absence of additional assumptions on $f$, it is easy to see that, in general $Z_{C}(\Gamma[f]) \leqq Z_{C}(f)$. But even if we impose on $f$ the additional restriction that all of its zeros lie in the left half-plane (i.e., have nonpositive real part), inequality (6) may still fail as the following example shows. 
EXAMPLE. Let $\Phi(x)=\sum_{k=0}^{\infty}\left(\gamma_{k} / k !\right) x^{k}=\left(x^{2}-1\right)^{2} \cos x$. Clearly, $\Phi$ is a function of type II in the Laguerre-Pólya class, and hence $\Gamma=$ $\left\{\gamma_{k}\right\}=\{1,0,-5,0,49,0, \cdots\}$ is a multiplier sequence of the second kind. If $f(x)=(x+1)^{2}\left(x^{2}+1\right)$, then $\Gamma[f(x)]=1-10 x^{2}+49 x^{4}$. Thus, $Z_{c}(\Gamma[f])=4$, while $Z_{c}(f)=2$.

3. Extensions and applications. This section is devoted to a brief treatment of some of the consequences of Theorem 1 and Theorem 3. (For different kinds of applications of Theorem 1, we refer the reader to [4].) As we shall see below, the principal leitmotif that underlies the various ramifications of inequality (6) is that this inequality serves as a unifying principle for many results of the type we cited in the Introduction.

Before we provide several generalizations of Theorem 1, we call attention to the following interesting partial converse of this theorem. If $h(x)=\sum_{k=0}^{n} b_{k} x^{k}$ is a real polynomial and if for all polynomials $f$

$$
Z_{C}\left(\sum_{k=0}^{n} b_{k} x^{k} f^{(k)}(x)\right) \leqq Z_{c}(f)
$$

then $h(x)$ has only real zeros. The proof of this assertion will be readily supplied by the reader.

Our first corollary shows that Theorem 1 remains valid if the polynomial $h(x)$ in this theorem is replaced by an entire function of type I in the Laguerre-Pólya class.

CoROLLARY 7. If $\Phi(x)=\sum\left(\gamma_{k} / k !\right) x^{k}, \gamma_{k} \geqq 0$, is a function of type I in the Laguerre-Pólya class and if $f(x)$ is an arbitrary real polynomial of degree $m$, then

$$
Z_{C}\left(\sum_{k=0}^{m} \frac{\gamma_{k}}{k !} x^{k} f^{(k)}(x)\right) \leqq Z_{C}(f) .
$$

Proof. Let $g_{n}(x)=\sum_{k=0}^{n}\left(\begin{array}{l}n \\ k\end{array}\right) \gamma_{k} x^{k}$ and apply Theorem 1 to $f(x)$ with $h(x)=g_{n}(x / n)$, and then consider the limit as $n \rightarrow \infty$.

We remark, parenthetically, that Corollary 7 extends a theorem of Pólya and Schur [20, p. 107]. It is interesting to note that this area of investigation is intimately connected with the various consequences of the Hermite-Poulain theorem [14, p. 238]. (For related theorems see also Obreschkoff [10] and [11].)

A companion result which generalizes a theorem of Pólya [14, p. 238] is the following corollary. 
CoROllaRY 8. Let $\Phi(x)=\sum_{k=0}^{\infty}\left(\gamma_{k} / k !\right) x^{k}, \gamma_{k} \geqq 0$, be a function of type I in the Laguerre-Pólya class. Let $\Psi_{1}(x)=\Psi(x) f(x)$, where $\Psi(x)$ is a function of type II in the Laguerre-Pólya class and $f$ is an arbitrary polynomial. Then

$$
Z_{C}\left(\sum_{k=0}^{\infty} \frac{\gamma_{k}}{k !} x^{k} \Psi_{1}^{(k)}(x)\right) \leqq Z_{C}(f)
$$

Proof. We first note that standard methods from the theory of entire functions show that the series

$$
\sum_{k=0}^{\infty} \frac{\gamma_{k}}{k !} x^{k} \Psi_{1}^{(k)}(x)
$$

converges in the whole plane. Let

$$
\begin{aligned}
& \Psi_{1}(x)=\sum_{k=0}^{\infty} \frac{\alpha_{k}}{k !} x^{k}, \\
& \psi_{n}(x)=\sum_{k=0}^{n}\left(\begin{array}{l}
n \\
k
\end{array}\right) \alpha_{k} x^{k}
\end{aligned}
$$

and

$$
g_{k}(x)=\sum_{j=0}^{k}\left(\begin{array}{l}
k \\
j
\end{array}\right) \gamma_{j} x^{j} .
$$

Then the sequence $\Lambda=\left\{g_{k}(1)\right\}_{k=0}^{\infty}$ is a multiplier sequence of the first kind (see the preliminary remarks in $\S 2$ ). Hence by Theorem 3 for each positive integer $n$

$$
Z_{C}\left(\Lambda\left[\psi_{n}(x)\right]\right) \leqq Z_{C}\left(\psi_{n}\right)
$$

Now by a result of Pólya [14, p. 246]

$$
Z_{c}\left(\psi_{n}\right) \leqq Z_{c}(f), \quad n=1,2, \cdots
$$

If we let $p_{n}(x)=\sum_{k=0}^{n}\left(\begin{array}{l}n \\ k\end{array}\right) g_{k}(1) \alpha_{k} x^{k}$, then the above inequalities imply that for each $n, Z_{c}\left(p_{n}\right) \leqq Z_{C}(f)$. Since the polynomials $p_{n}(x / n)$ converge uniformly on compact subsets of the plane to the entire function

$$
\sum_{k=0}^{\infty} g_{k}(1) \frac{\alpha_{k}}{k !} x^{k}
$$

we conclude that

$$
Z_{C}\left(\sum_{k=0}^{\infty} g_{k}(1) \frac{\alpha_{k}}{k !} x^{k}\right) \leqq Z_{C}(f)
$$


But now a calculation shows that

$$
\sum_{k=0}^{\infty} g_{k}(1) \frac{\alpha_{k}}{k !} x^{k}=\sum_{k=0}^{\infty} \frac{\gamma_{k}}{k !} x^{k} \Psi_{1}^{(k)}(x),
$$

and thus the conclusion of the corollary follows.

The same type of argument establishes, mutatis mutandis, the validity of our next corollary.

CoRollary 9. If $\Gamma=\left\{\gamma_{k}\right\}_{k=0}^{\infty}, \gamma_{k} \geqq 0$, is a multiplier sequence of the first kind and if $\Psi(x)$ is a function of type II in the Laguerre-Pólya class, then for any real polynomial $f(x)$

$$
Z_{C}(\Gamma[\Psi(x) f(x)]) \leqq Z_{C}(f)
$$

We pause here for a moment to examine the scope of the foregoing results. Let $\Gamma^{(0)}=\{1,1,1, \cdots\}, \Gamma^{(1)}=\{0,1,2, \cdots\}$ and for a positive integer $m, m \geqq 2$, let $\Gamma^{(m)}=\left\{\gamma_{k}\right\}_{k=0}^{\infty}$, where $\gamma_{0}=\gamma_{1}=\cdots=$ $\gamma_{m-1}=0$ and $\gamma_{m+k}=(m+k) ! / k !, k=0,1,2, \cdots$. Then for each nonnegative integer $m, \Gamma^{(m)}$ is a multiplier sequence of the first kind (just consider the Taylor coefficients of the function $x^{m} e^{x}$ ) and $\Gamma^{(m)}$ is the multiplier sequence which corresponds to the operator $D^{m}$, where $D=d / d x$. More precisely if $\Phi$ is an entire function, then

$$
x^{m} D^{m} \Phi=\Gamma^{(m)}[\Phi] .
$$

Thus, Corollary 9 asserts in this case that $Z_{C}\left(\Gamma^{(m)}[\Psi(x) f(x)]\right) \leqq Z_{C}(f)$, where $\Psi$ is a function of type II in the Laguerre-Pólya class and $f$ is an arbitrary real polynomial. Now a fifty-year old conjecture (see, for example, Pólya [17], [18] and [19] and Wiman [26]) asserts, when expressed in our nomenclature, that there is a positive integer $m$, sufficiently large, such that

$$
Z_{C}\left(\Gamma^{(m)}[\Psi(x) f(x)]\right)=0
$$

Thus, this conjecture may be viewed as a special case of the more general problem we referred to in the discussion preceding Theorem 5 .

We shall mention here a consequence of Corollary 9 which generalizes Theorem 3 and a theorem of Pólya [14] in yet a different direction.

COROLlaRY 10. If $\Phi(x)=\sum_{k=0}^{\infty}\left(\gamma_{k} / k !\right) x^{k}, \gamma_{k} \geqq 0$, is a function of type I in the Laguerre-Pólya class and if $f(x)=\sum_{k=0}^{n} a_{k} x^{k}$ is an arbitrary polynomial, then for any fixed real number $t$ 


$$
Z_{C}\left(\sum_{k=0}^{n} a_{k} x^{k} \Phi^{(k)}(x t)\right) \leqq Z_{C}(f)
$$

Proof. Let $\Gamma=\left\{\gamma_{k}\right\}_{k=0}^{\infty}$, so that $\Gamma$ is a multiplier sequence of the first kind. Then a computation shows that

$$
\Gamma\left[e^{x t} f(x)\right]=\sum_{k=0}^{n} a_{k} x^{k} \Phi^{(k)}(x t) .
$$

Hence by Corollary 9 for each fixed $t$

$$
Z_{c}\left(\sum_{k=0}^{n} a_{k} x^{k} \Phi^{(k)}(x t)\right) \leqq Z_{c}(f) .
$$

It is instructive to note that in the special case when $t=0$, Corollary 10 reduces to Theorem 3 .

We shall now briefly describe the connection between Laguerre's theorem and Theorem 1 and Theorem 3. Let $h(x)=\sum_{k=0}^{n} b_{k} x^{k}$ be a real polynomial with only real negative zeros. Let $f(x)=\sum_{k=0}^{m} a_{k} x^{k}$ be a real polynomial and let $\theta=x(d / d x)$. Then

$$
h(\theta) f=\sum_{k=0}^{m} a_{k} h(k) x^{k},
$$

and by Laguerre's theorem

$$
Z_{c}(h(\theta) f) \leqq Z_{c}(f) .
$$

Thus, the sequence $\{h(k)\}_{k=0}^{\infty}$ is a multiplier sequence of the first kind. In light of Theorem 3 it is clear now that the fundamental inequality is an extension of Laguerre's theorem (for other kinds of extensions of Laguerre's theorem, see [16]). Indeed, there are real polynomials $p(x)$, not all of whose zeros are real, for which the sequence $\{p(k)\}_{k=0}^{\infty}$ is a multiplier sequence of the first kind (consider, for example, $\left.p(x)=1+x+x^{2}\right)$. The next corollary provides a method for constructing multiplier sequences of the form just mentioned.

CoROLlary 11. Let $h(x)=\sum_{k=0}^{n} b_{k} x^{k}$ be a real polynomial with only real negative zeros. Let $\tilde{h}(x)$ denote the polynomial

$$
\widetilde{h}(x)=\sum_{k=0}^{n} b_{k} x(x-1) \cdots(x-k+1) .
$$

If $f(x)=\sum_{k=0}^{m} a_{k} x^{k}$ is an arbitray real polynomial, then

$$
Z_{C}\left(\sum_{k=0}^{m} a_{k} \widetilde{h}(k) x^{k}\right) \leqq Z_{C}(f) .
$$


In particular, the sequence $\{\tilde{h}(k)\}_{k=0}^{\infty}$ is a multiplier sequence of the first kind.

Proof. If $\theta=x(d / d x)$, then an easy induction shows that $\theta(\theta-1) \cdots(\theta-k+1) f=x^{k} f^{(k)}(x)$. Hence

$$
\widetilde{h}(\theta) f=\sum_{j=0}^{n} b_{j} x^{j} f^{(j)}(x) .
$$

Since by assumption $h(x)$ has only real negative zeros, we may invoke Theorem 1 and deduce that

$$
Z_{C}(\widetilde{h}(\theta) f) \leqq Z_{c}(f)
$$

On the other hand,

$$
\begin{aligned}
\widetilde{h}(\theta) f & =\sum_{j=0}^{n} b_{j} x^{j} f^{(j)}(x) \\
& =\sum_{j=0}^{n} b_{j} \sum_{k=j}^{m} a_{k} \frac{k !}{(k-j) !} x^{k} \\
& =\sum_{k=0}^{m} a_{k} \sum_{j=0}^{k} b_{j} k(k-1) \cdots(k-j+1) x^{k} \\
& =\sum_{k=0}^{m} a_{k} \widetilde{h}(k) x^{k} .
\end{aligned}
$$

Consequently,

$$
Z_{C}\left(\sum_{k=0}^{m} a_{k} \tilde{h}(k) x^{k}\right) \leqq Z_{c}(f)
$$

The observations introduced in the course of the proof of Corollary 11 allow us to reformulate Theorem 1 in terms of the differential operator $\theta$ and the polynomial $\tilde{h}(x)$, where $h(x)$ has only real negative zeros. That is, the inequality in Theorem 1 may now be written as

$$
Z_{G}(\tilde{h}(\theta) f) \leqq Z_{C}(f) .
$$

We hasten to add that the polynomial $\tilde{h}(x)$ need not have any real zeros even if all the zeros of $h(x)$ are real and negative (set $h(x)=$ $\left.(1+x)^{2}\right)$. However, if $h(x)$ has only real positive zeros, then it is known [21, V, $\# 185]$ that $\bar{h}(x)$ has also only real positive zeros. For related results about polynomials of the form $\widetilde{h}(x)$, where $h(x)$ is an arbitrary real polynomial, we refer the reader to Pólya and Szegö [21, V, \#182-188] and Obreschkoff [12].

The remarkable properties of multiplier sequences were first derived from the Schur Composition Theorem [22], [9], [12] and [20]. Thus, in light of the foregoing developments, it is not sur- 
prising that the fundamental inequality also implies the following extension of the Schur Composition Theorem.

Corollary 12. Let $h(x)=\sum_{k=0}^{n} b_{k} x^{k}, b_{n} \neq 0$, be a real polynomial with only real negative zeros and let $f(x)=\sum_{k=0}^{n} a_{k} x^{k}, a_{n} \neq 0$, be an arbitrary real polynomial. Then

$$
Z_{C}\left(\sum_{k=0}^{n} a_{k} b_{k} x^{k}\right) \leqq Z_{C}(f)
$$

Proof. If we write $h(x)=\sum_{k=0}^{n} k !\left(b_{k} / k !\right) x^{k}$, then it follows from the transcendental characterization of multiplier sequences of the first kind (see $\S 2$ ), that the sequence $\Gamma=\left\{k ! b_{k}\right\}_{k=0}^{\infty}$ is a multiplier sequence of the first kind. Hence by Theorem 3

$$
Z_{C}(\Gamma[f])=Z_{c}\left(\sum_{k=0}^{n} k ! b_{k} a_{k} x^{k}\right) \leqq Z_{C}(f)
$$

Since the sequence $\{1 / k !\}$ is a multiplier sequence of the first kind, it follows once again from Theorem 3 that

$$
Z_{C}\left(\sum_{k=0}^{n} b_{k} a_{k} x^{k}\right) \leqq Z_{C}(f)
$$

The various composition theorems of Grace [12], De Bruijn and Springer [6], Marden [8], Obreschkoff [12], Szegö [24] and Weisner [25] just to mention a few, belong to the same circle of ideas that we have been investigating in this paper. (A clear account of these results is given in Marden [9, Chapter IV]; see also Obreschkoff [12, Chapter II].) However, these beautiful geometric theorems treat, for the most part, only the location of the nonreal zeros. In contrast, our results give information on the number of nonreal zeros of the composite polynomials. It is in this sense that the fundamental inequality and its consequences supplement the existing knowledge in the theory of distribution of zeros of polynomials and entire functions.

In this short list of direct consequences of Theorem 3, we shall also include a Stieltjes integral representation of $\Gamma[f]$, since it leads to an interesting open problem.

CoRollary 13. Let $\Gamma=\left\{\gamma_{k}\right\}, \gamma_{0} \neq 0$, be a multiplier sequence of the first kind. Then there is a function $\beta(t)$ of bounded variation on $(0, \infty)$ with the following properties:

(a) The moment constants 


$$
\gamma_{k}=\int_{0}^{\infty} t^{k} d \beta(t)
$$

all exist.

(b) For any polynomial $f$,

$$
\Gamma[f]=\int_{0}^{\infty} f(x t) d \beta(t) .
$$

In particular

$$
Z_{C}\left(\int_{0}^{\infty} f(x t) d \beta(t)\right) \leqq Z_{C}(f)
$$

Since the polynomial set $\left\{g_{k}^{*}(x) / k !\right\}_{k=0}^{\infty}$, where $g_{k}^{*}(x)=\left(\Gamma\left[1+x^{k}\right]\right)^{*}$, is an Appell set, Corollary 13 is a direct consequence of well-known results (see, for example, Sheffer [23]) and Theorem 3.

The open problem we alluded to may be formulated as follows. Characterize the measures $d \beta$ for which the inequality (b) of Corollary 13 holds for all real polynomials $f$.

Thus far we have witnessed several similarities between the linear operators $\Gamma$ and $D, D=d / d x$. In conclusion, we shall cite two results which further elucidate the relationship between these operators. In the formulation of Corollary 14, we require the following additional terminology. Let $\Gamma=\left\{\gamma_{k}\right\}_{k=0}^{\infty}$ be a multiplier sequence of the first kind. By a shift of $\Gamma$, we mean the sequence $\left\{\gamma_{m}, \gamma_{m+1}, \gamma_{m+2}, \cdots\right\}$ for some nonnegative integer $m$. The $m$ th shift of $\Gamma$ will be denoted by $\Gamma_{m}=\left\{\gamma_{m+k}\right\}_{k=0}^{\infty}, \Gamma_{0}=\Gamma$. It is easy to see that $\Gamma_{m}$ is also a multiplier requence of the first kind. In the following two corollaries, the assumption that $\gamma_{k}>0, k=0,1,2, \cdots$, is essential.

Corollary 14 (The Hermite-Poulain Theorem). Let $h(x)=$ $\sum_{k=0}^{n} b_{k} x^{k}, b_{n}=1$, be a real polynomial with only real negative zeros. Let $f$ be an arbitrary real polynomial and let $\Gamma=\left\{\gamma_{k}\right\}, \gamma_{k}>0$, be a multiplier sequence of the first kind. Define

$$
\varphi(x)=\sum_{k=0}^{n} b_{k} \Gamma_{k}[f(x)]
$$

and

$$
\psi(x)=\sum_{k=0}^{n} b_{k} x^{k} \Gamma_{k}[f(x)]
$$

where $\Gamma_{k}$ denotes the kth shift of $\Gamma$. Then (a) $Z_{C}(\varphi) \leqq Z_{C}(f)$ and (b) $Z_{C}(\psi) \leqq Z_{C}(f)$.

Proof. Let $h(x)=\left(x+\alpha_{n}\right) \cdots\left(x+\alpha_{1}\right)$, where $\alpha_{j}>0$, and form 
the polynomial

$$
F(x)=\alpha_{1} \Gamma[f(x)]+\Gamma_{1}[f(x)],
$$

where $\Gamma_{1}$ is the first shift of $\Gamma$. Let $\Lambda=\alpha_{1} \Gamma+\Gamma_{1}$ so that $\Lambda=$ $\left\{\alpha_{1} \gamma_{k}+\gamma_{k+1}\right\}_{k=0}^{\infty}$. Since $\alpha_{1}>0$, it follows from the classical HermitePoulain theorem [12, p. 4], [14, p. 238] and a theorem of Pólya and Schur [20, p. 110] that $\Lambda$ is a multiplier sequence of the first kind. Hence by Theorem 3

$$
Z_{C}(F)=Z_{C}(\Lambda[f]) \leqq Z_{C}(f) .
$$

Next we form the sequence $\alpha_{2} \Lambda+\Lambda_{1}$, where $\Lambda_{1}$ denotes the first shift of $\Lambda$. As before we obtain

$$
Z_{c}\left(\left(\alpha_{2} \Lambda+\Lambda_{1}\right)[f]\right) \leqq Z_{c}(f) .
$$

Repeated applications of the above argument yield $Z_{C}(\varphi) \leqq Z_{C}(f)$, and thus we have proved part (a) of the corollary. Part (b) of the corollary is an immediate consequence of Theorem 3.

It is instructive to note that if in the above definitions of the polynomials $\varphi$ and $\psi$ we replace $\Gamma_{k}$ by $D^{k}$, where $D^{k}=d^{k} / d x^{k}$, then part (a) of Corollary 14 reduces to the classical Hermite-Poulain theorem, while part (b) becomes the fundamental inequality with the multiplier sequence $\{\tilde{h}(k)\}_{k=0}^{\infty}$.

CoRollary 1.5. Let $\Gamma=\left\{\gamma_{k}\right\}, \gamma_{k}>0, k=0,1,2, \cdots$, be $a$ multiplier sequence of the first kind and let $f$ be an arbitrary real polynomial. Suppose that $Z_{C}(\Gamma[f])=Z_{C}(f)$. If $\Gamma[f]$ possesses a multiple real zero, then so does $f$.

Proof. If $f$ does not have a multiple real zero, then $Z_{C}(f)=$ $Z_{c}(f+\varepsilon)$ for $|\varepsilon|$ sufficiently small. But then with the appropriate sign for $\varepsilon, \Gamma[f+\varepsilon]=\Gamma[f]+\gamma_{0} \varepsilon$ will have more nonreal zeros than $\Gamma[f]$. That is,

$$
Z_{c}(\Gamma[f+\varepsilon])>Z_{c}(\Gamma[f])=Z_{c}(f)=Z_{c}(f+\varepsilon) .
$$

This contradicts the fundamental inequality and hence the proof of the corollary is complete.

\section{REFERENCES}

1. R. P. Boas, Jr., George Pólya: Collected Papers, vol. 2, M. I. T. Press, Cambridge, Massachusetts, 1974.

2. T. Craven and G. Csordas, Multiplier sequences for fields, Illinois J. Math., 21 (1977), 801-817. 
3. T. Craven and G. Csordas, On the number of real roots of polynomials, to appear. 4. - Zero-diminishing linear transformations, Proc. Amer. Math. Soc., 80 (1980), 544-546.

5. G. Csordas and J. Williamson, The zeros of Jensen polynomials are simple, Proc. Amer. Math. Soc., 49 (1975), 263-264.

6. N.G. De Bruijn and T.A. Springer, On the zeros of composition polynomials, Nederl. Akad. Wetensch. Proc., 50 (1947), 895-903.

7. B. Ja. Levin, Distribution of Zeros of Entire Functions, Amer. Math. Soc. Transl., Providence, Rhode Island, 1964.

8. M. Marden, The zeros of certain composite polynomials, Bull. Amer. Math. Soc., 49 (1943), 93-100.

9. - Geometry of Polynomials, Math. Surveys no. 3, Amer. Math. Soc., Providence, Rhode Island, 1966.

10. N. Obreschkoff, Sur le théorème de Hermite et Poulain, C. R. Acad. Sci. Paris, 249 (1959), 21-22.

11. - Sur une généralization du théorème de Poulain et Hermite pour les zéros réels des polynomes réels, Acta Math. Acad. Sci. Hungar., 12 (1961), 175-184. (Russian summary).

12. $\longrightarrow$ Verteilung und Berechnung der Nullstellen Reeler Polynome, Veb Deutscher Verlag der Wissenschaften, Berlin, 1963.

13. G. Pólya, Über Annäherung durch Polynome mit Lauter reellen Wurzeln, Rend. Circ. Mat. Palermo., 36 (1913), 279-295.

14. - Algebraische Untersuchungen über ganze Funktionen von Geschlechte Null und Eins, J. Reine Angew. Math., 145 (1914), 224-249.

15. — Über algebraische Gleichungen mit nur reelen Wurzeln, Vierteljschr. Naturforsch. Ges. Zurich, 61 (1916), 546-548.

16. — Über einen Satz von Laguerre, Jber. Deutsch. Math. Verein., 38 (1929), 161-168.

17. - Some problems connected with Fourier's work on transcendental questions, Quart. J. Math. Oxford, Ser., 1 (1930), 21-34.

18. — Ü Über die Realität der Nullstellen fast aller Ableitungen gewisser ganzer Funktionen, Math. Ann., 114 (1937), 622-634.

19. - On the zeros of the derivatives of a function and its analytic character, Bull. Amer. Math. Soc., 49 (1943), 178-191.

20. G. Pólya and J. Schur, Über zwei Arten von Faktorenfolgen in der Theorie der algebraischen Gleichungen, J. Reine Angew. Math., 144 (1914), 89-113.

21. G. Pólya and G. Szegö, Autgaben und Lehrsätze aus der Analysis, vol. II, Springer-Verlag, Berlin, 1971.

22. J. Schur, Zwei Sätze über algebraische Gleichungen mit lauter reellen Wurzeln, J. Reine Angew. Math., 144 (1914), 75-88.

23. I. M. Sheffer, Note on Appell polynomials, Bull. Amer. Math. Soc., 51 (1945), 739-744.

24. G. Szegö, Bemerkungen zu einem Satz von J. H. Grace über die Wurzeln algebraischer Gleichungen, Math. Z., 13 (1922), 28-55.

25. L. Weisner, Roots of certain classes of polynomials, Bull. Amer. Math. Soc., 48 (1942), 283-286.

26. A. Wiman, Über die Realität der Nullstellen fast aller Ableitungen gewisser ganzer Funktionen, Math. Ann., 114 (1937), 617-621.

Received January 29, 1980. The first author was partially supported by NSF grant MCS79-00318.

UNIVERSITY OF HAWAII

HoNOLULU HI 96822 


\section{PACIFIC JOURNAL OF MATHEMATICS}

\section{EDITORS}

DONALD BABBITT (Managing Editor)

University of California

Los Angeles, CA 90024

HUGo RossI

University of Utah

Salt Lake City, UT 84112

C. C. MOORE and ANDREW OGG

University of California

Berkeley, CA 94720
J. DugundjI

Department of Mathematics

University of Southern California

Los Angeles, CA 90007

R. FinN and J. Milgram

Stanford University

Stanford, CA 94305

\section{ASSOCIATE EDITORS}
R. ARENS
E. F. BECKENBACH
B. H. NeumanN
F. WOLF
K. YOSHIDA

\section{SUPPORTING INSTITUTIONS}

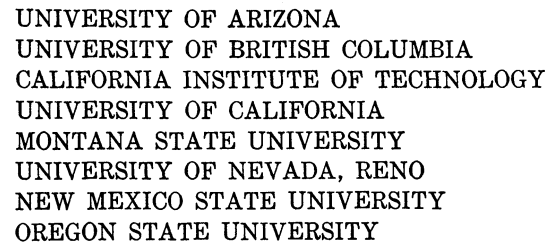

UNIVERSITY OF ARIZONA

UNIVERSITY OF BRITISH COLUMBIA

CALIFORNIA INSTITUTE OF TECHNOLOGY

UNIVERSITY OF CALIFORNIA

MONTANA STATE UNIVERSITY

UNIVERSITY OF NEVADA, RENO

NEW MEXICO STATE UNIVERSITY

OREGON STATE UNIVERSITY

\author{
UNIVERSITY OF OREGON \\ UNIVERSITY OF SOUTHERN CALIFORNIA \\ STANFORD UNIVERSITY \\ UNIVERSITY OF HAWAII \\ UNIVERSITY OF TOKYO \\ UNIVERSITY OF UTAH \\ WASHINGTON STATE UNIVERSITY \\ UNIVERSITY OF WASHINGTON
}

The Supporting Institutions listed above contribute to the cost of publication of this Journal, but they are not owners or publishers and have no responsibility for its content or policies.

Mathematical papers intended for publication in the Pacific Journal of Mathematics should be in typed form or offset-reproduced, (not dittoed), double spaced with large margins. Please do not use built up fractions in the text of the manuscript. However, you may use them in the displayed equations. Underline Greek letters in red, German in green, and script in blue. The first paragraph or two must be capable of being used separately as a synopsis of the entire paper. Please propose a heading for the odd numbered pages of less than 35 characters. Manuscripts, in triplicate, may be sent to any one of the editors. Please classify according to the scheme of Math. Reviews, Index to Vol. 39. Supply name and address of author to whom proofs should be sent. All other communications should be addressed to the managing editor, or Elaine Barth, University of California, Los Angeles, California, 90024.

50 reprints to each author are provided free for each article, only if page charges have been substantially paid. Additional copies may be obtained at cost in multiples of 50 .

The Pacific Journal of Mathematics is issued monthly as of January 1966. Regular subscription rate: $\$ 102.00$ a year (6 Vols., 12 issues). Special rate: $\$ 51.00$ a year to individual members of supporting institutions.

Subscriptions, orders for numbers issued in the last three calendar years, and changes of address shoud be sent to Pacific Journal of Mathematics, P.O. Box 969, Carmel Valley, CA 93924, U.S.A. Old back numbers obtainable from Kraus Per!odicals Co., Route 100, Millwood, NY 10546.

PUBLISHED BY PACIFIC JOURNAL OF MATHEMATICS, A NON-PROFIT CORPORATION

Printed at Kokusai Bunken Insatsusha (International Academic Printing Co., Ltd.). 8-8, 3-chome, Takadanobaba, Shinjuku-ku, Tokyo 160, Japan. 


\section{Pacific Journal of Mathematics}

\section{Vol. 95, No. $2 \quad$ October, 1981}

George E. Andrews, The Rogers-Ramanujan reciprocal and Minc's

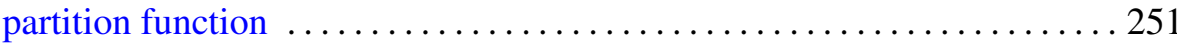

Allan Calder, William H. Julian, Ray Mines, III and Fred Richman,

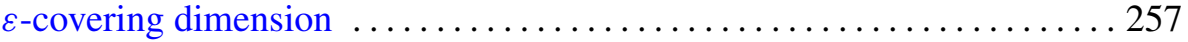

Thomas Curtis Craven and George Leslie Csordas, An inequality for the distribution of zeros of polynomials and entire functions $\ldots \ldots \ldots \ldots 263$

Thomas Jones Enright and R. Parthasarathy, The transfer of invariant

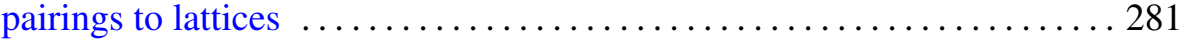

Allen Roy Freedman and John Joseph Sember, Densities and

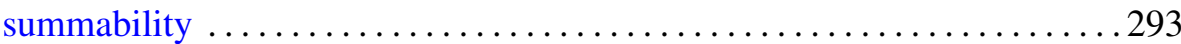

Robert Heller and Francis Aubra Roach, A generalization of a classical necessary condition for convergence of continued fractions . . . . . . 307

Peter Wilcox Jones, Ratios of interpolating Blaschke products ........... 311

V. J. Joseph, Smooth actions of the circle group on exotic spheres ........ 323

Mohd Saeed Khan, Common fixed point theorems for multivalued

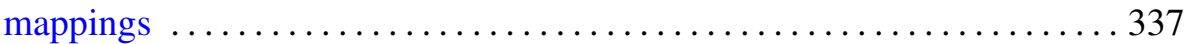

Samuel James Lomonaco, Jr., The homotopy groups of knots. I. How to

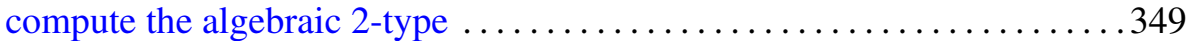

Louis Magnin, Some remarks about $C^{\infty}$ vectors in representations of connected locally compact groups ............................ 391

Mark Mandelker, Located sets on the line . . . . . . . . . . . . . . . . . 401

Murray Angus Marshall and Joseph Lewis Yucas, Linked quaternionic mappings and their associated Witt rings $\ldots \ldots \ldots \ldots \ldots \ldots \ldots \ldots . \ldots \ldots 11$

William Lindall Paschke, $K$-theory for commutants in the Calkin algebra

W. J. Phillips, On the relation $P Q-Q P=-i I$ 435

Ellen Elizabeth Reed, A class of Wallman-type extension. 443

Sungwoo Suh, The space of real parts of algebras of Fourier transforms 461 Antonius Johannes Van Haagen, Finite signed measures on function

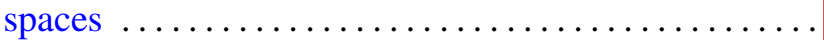

Richard Hawks Warren, Identification spaces and unique uniformity 